\title{
Manganese Catalyzed Reformation of Ethylene Glycol to Glycolic acid and Lactic Acid
}

\author{
Satyadeep Waiba, Biplab Maji* \\ Department of Chemical Sciences, Indian Institute of Science Education and Research Kolkata, Mohanpur, 741246, India. \\ Manganese catalysis, Dehydrogenative coupling, Lactic Acid, Glycolic acid, Reformation, Sustainable synthesis, Hydrogen
}

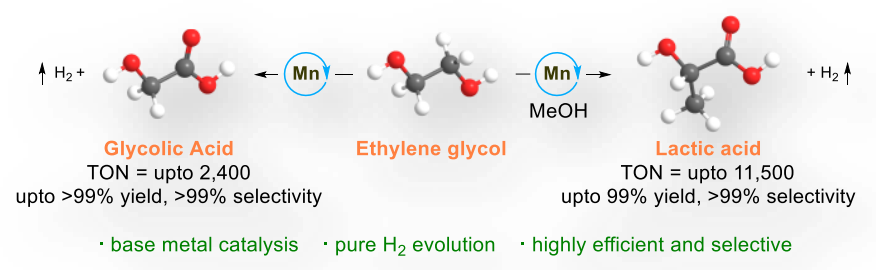

\begin{abstract}
Conversion of readily available feedstocks to valuable platform chemicals via a sustainable catalytic pathway has always been one of the key focuses of synthetic chemists. Cheaper, less toxic, and more abundant base metals as a catalyst for performing such transformations provide an additional boost. In this context, herein, we report a reformation of readily available feedstock, ethylene glycol, to value-added platform molecules, glycolic acid, and lactic acid. A bench stable base metal complex $\left\{\left[\mathrm{HN}\left(\mathrm{C}_{2} \mathrm{H}_{4} \mathrm{PPh}_{2}\right)_{2}\right] \mathrm{Mn}(\mathrm{CO})_{2} \mathrm{Br}\right\}, \mathbf{M n}-\mathbf{I}$, known as $\mathrm{Mn}-{ }^{\mathrm{Ph}} \mathrm{MACHO}$, catalyzed the reformation of ethylene glycol to glycolic acid at 140 ${ }^{\circ} \mathrm{C}$ in high selectivity with a turnover number TON $=2400$, surpassing previously used homogeneous catalysts for such a reaction. Pure hydrogen gas is evolved without the need for an acceptor. On the other hand, a bench stable Mn(I)-complex, $\left\{\left({ }^{i \mathrm{Pr}} \mathrm{PN}{ }^{5} \mathrm{P}\right) \mathrm{Mn}(\mathrm{CO})_{2} \mathrm{Br}\right\}, \mathrm{Mn}-\mathrm{III}$, with a triazine backbone, efficiently catalyzed the acceptorless dehydrogenative coupling of ethylene glycol and methanol for the synthesis of lactic acid, even at a ppm level of catalyst loading, reaching the TON of 11,500. Detailed mechanistic studies were performed to elucidate the involvements of different manganese(I)-species during the catalysis.
\end{abstract}

\section{INTRODUCTION}

Converting widely accessible renewable feedstock into valueadded products and alternative fuel sources is highly relevant in terms of synthetic output and sustainable chemical development. ${ }^{1-8}$ In this context, ethylene glycol (EG) is readily available and cheaply sourced, both from biomass and fossil fuel-based resources, and is an excellent feedstock for such derivatization (Figure 1A). ${ }^{9-10}$ Industrially, EG has been utilized as a coolant, heat-transfer, anti-freeze agent, and precursor for blockbuster polymers. ${ }^{9-10}$ Recently, EG has also emerged as a promising candidate for hydrogen gas carriers with a theoretical hydrogen storage capacity (HSC) of $6.5 \mathrm{wt} \% .{ }^{11}$ Consequently, it has been considered a viable candidate for serving as a liquid organic hydrogen carrier (LOHC). On the contrary, the valorization of EG for synthesizing various value-added products is relatively finite.

Glycolic acid (GA) and lactic acid (LA), having both hydroxy and acid functional groups, are highly demanded with widespread applications ranging from food, skincare, cosmetics, pharmaceuticals, textile, and polymer (Figure 1A). ${ }^{12-15}$ Furthermore, they are widely used to produce various green solvents and commodity chemicals. ${ }^{14}$ More intriguingly, both these acids are precursors of widely used biodegradable and biocompatible polymer polyglycolic acid (PGA) and polylactic acid (PLA). ${ }^{16-}$

${ }^{17}$ Owing to their diverse applications, the global market for LA in 2022 is estimated to cross $4 \times 10^{5}$ tons per year, and for GA to exceed 400 million USD by $2024 .{ }^{14,18}$ While the current manufacturing protocol for LA is based on conventional chemocatalysis and fermentative methods, the current production of GA largely depends on high-pressure formaldehyde carbonylation using $\mathrm{CO}$ at cryogenic conditions, or hydrolysis of chloroacetic acid or methyl 2-hydroxyacetate, cyanidation, transesterification, and enzymatic reaction. ${ }^{19-21}$ Although used industrially, these reactions suffer from major sustainability issues like stoichiometric waste generation, multi-step purification, separation, and toxic reagents requirement. Alternatively, an environmentally benign direct conversion of readily available renewable biomass, like EG, to GA and LA under mild and atom economic conditions is valued as highly desirable. ${ }^{21-25}$ However, it is less explored to date.

Contextually, Dumesic demonstrated the first reformation of aqueous $\mathrm{EG}$ to $\mathrm{H}_{2}, \mathrm{CO}_{2}$, and short alkanes at elevated temperatures using $\mathrm{Pt} / \mathrm{Al}_{2} \mathrm{O}_{3}$ and Raney-NiSn (Figure 1B). ${ }^{26,27}$ Bitter attempted carbon nanofiber $(\mathrm{CNF})$ supported $\mathrm{Cu}$, and Ni nanoparticles promoted conversion of $\mathrm{EG}$ to $\mathrm{GA}$ and $\mathrm{H}_{2} \cdot{ }^{23}$ However, further decomposition of GA to formic acid, $\mathrm{H}_{2}$, and $\mathrm{CO}_{2}$ was observed. Independently, Cole-Hamilton ${ }^{28}$ and Beller ${ }^{29}$ disclosed the reformation of $\mathrm{EG}$ to $\mathrm{H}_{2}$; however, the formation of GA was not confirmed. A homogeneous Rh-catalyzed oxidative dehydrogenation of alcohols and polyalcohols, including EG, to carboxylic acids in the presence of sacrificial hydrogen acceptors such as ketones or methyl methacrylate was developed. ${ }^{22}$ 
Figure 1 (A) Source and Usage of Ethylene Glycol. (B) Previous Reformation of Ethylene Glycol and Lactic Acid Synthesis. (C) Manganese Catalyzed Reformation of Ethylene Glycol to Glycolic acid and Lactic Acid.

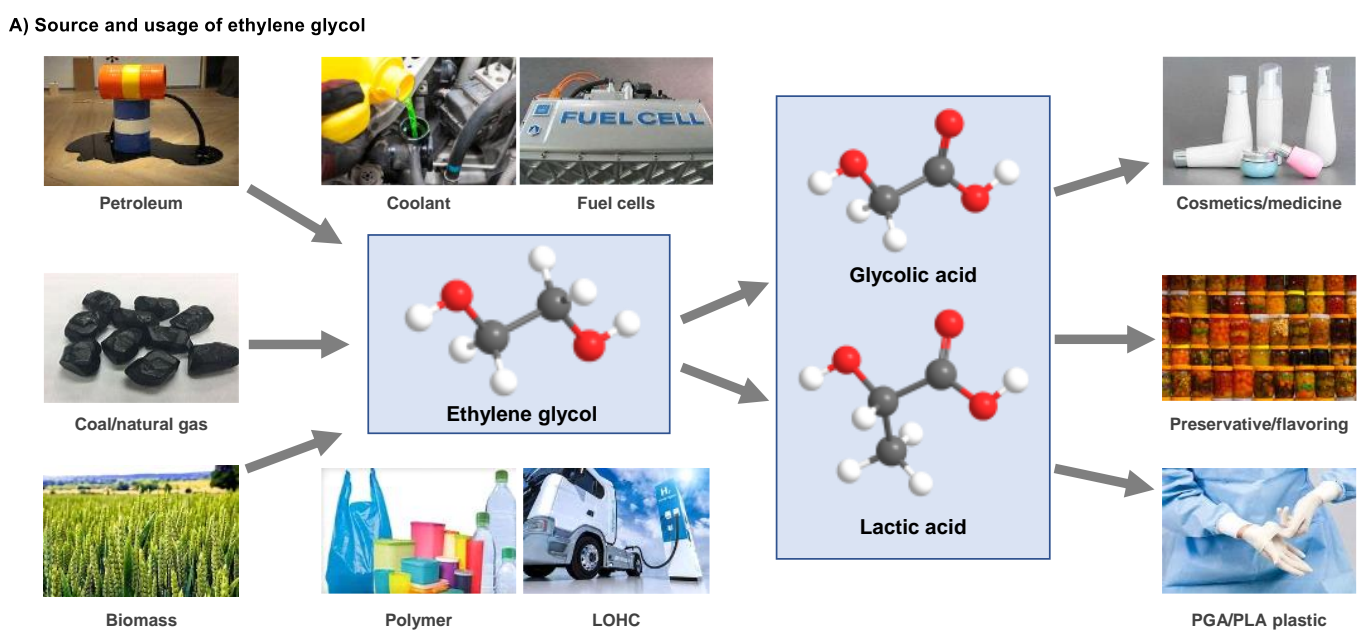

B) Previous reformation of ethylene glycol and chemical synthesis of lactic acid

Reformation of ethylene glycol

i) Reformation of EG to hydrogen:

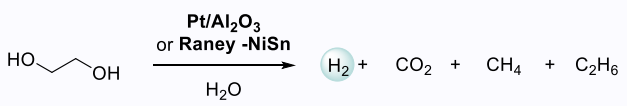

ii) Heterogeneous reformation of EG to glycolic acid:

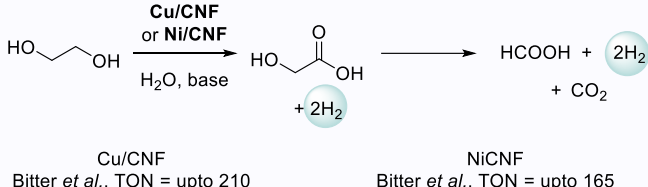

iii) Homonogeneous Ir- and Ru-catalyzed transformation of EG to GA:

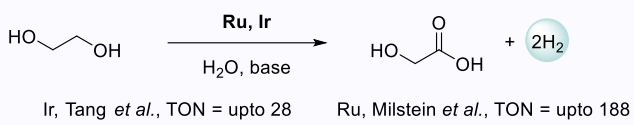

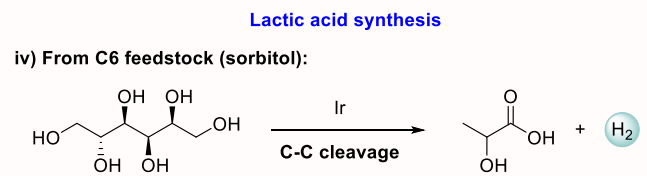

Ir, Crabtree et al. TON $=$ upto 400

v) From C3 feedstock (glycerol):

$$
\begin{aligned}
& \underbrace{\mathrm{Ir}, \mathrm{Ru}, \mathrm{Fe}}_{\mathrm{OH}} \longrightarrow \mathrm{OH}_{\mathrm{OH}}^{\mathrm{OH}}+\mathrm{H}_{2} \\
& \begin{array}{ccc}
\text { Ir, Crabtree et al., } & \text { Ir, Williams et al., } & \text { Ir, Tu et al., } \\
\text { TON }=\text { upto } 3.01 \times 10^{4} & \text { TON = upto } 4.56 \times 10^{6} & \text { TON = upto } 1.24 \times 10^{5}
\end{array} \\
& \mathrm{Ru}, \text { Beller et al., Fe, Crabtree et al. } \\
& \begin{array}{ll}
\text { TON }=\text { upto } 2.65 \times 10^{5} & \text { TON = upto } 1.050
\end{array}
\end{aligned}
$$

vi) From $\mathrm{C} 2$ and $\mathrm{C} 1$ (EG and $\mathrm{MeOH}$ ):

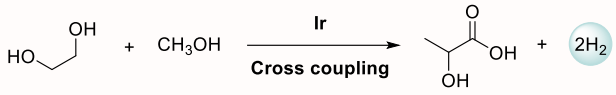

Ir, Tu et al., TON $=$ upto 40,000

C) This work: Base metal catalyzed reformation of ethylene glycol to glycolic acid and lactic acid

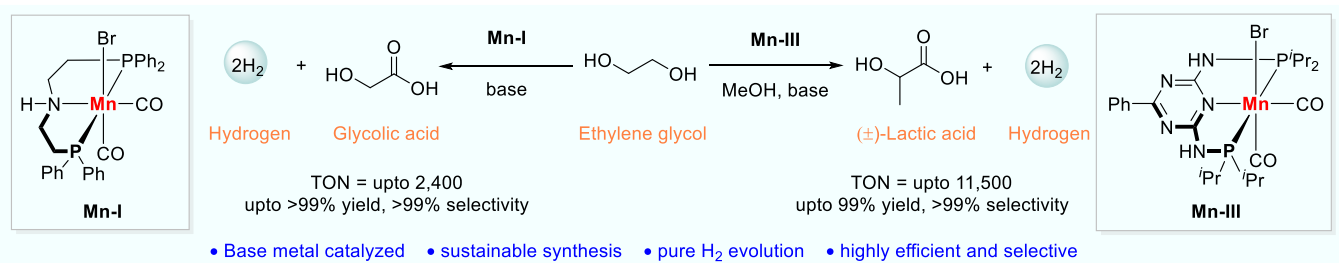

Recently, homogeneous iridium- (turn over number, TON up to 28 ) and ruthenium-catalyzed (TON up to 188) reformation of EG to GA were independently developed by Tang, ${ }^{24}$ and Milstein, ${ }^{21}$ respectively (Figure 1B). However, the need for sacrificial hydrogen acceptor, scarce noble metals, poor selectivity, and low TON somewhat limits the scope of these reactions.

Contemporarily, the reconstruction of C6 (sorbitol) ${ }^{30}$ and C3 (glycerol) $^{29,31-34}$ sources had offered an appealing chemical route for the synthesis of LA (Figure 1B). Nonetheless, low selectivity due to undesired $\mathrm{C}-\mathrm{C}$ bond cleavage and low atom economy remain the key issues. As an alternative, a dehydrogenative coupling (DHC) between EG and $\mathrm{MeOH}$ can be carried out to synthesize this platform molecule. The protocol, however, comes with the challenge that the catalyst needs to be highly selective for $\mathrm{EG}$ and $\mathrm{MeOH}$ dehydrogenation and cross-coupling of in situ generated glycolaldehyde and formaldehyde. At the same time, the unproductive homo-coupling of aldehydes, overoxidation, esterification, or polymerization of EG must be suppressed. To the best of our knowledge, only one iridium- $N$-heterocyclic carbene complex successfully realized the DHC of EG and $\mathrm{MeOH}$ to LA in high yields and selectivities (Figure 1B). ${ }^{25}$ Nevertheless, the synthesis of LA and also GA from readily available natural resources utilizing non-noble metal catalysts with high yield and selectivity is the need of the hour. ${ }^{14,21-24}$

Recently, molecularly defined complexes of the third most abundant metal manganese have emerged as a powerful 
catalyst for various hydrogen transfer reactions. ${ }^{35-43}$ In this aspect, several groups, including ours, have also been involved in developing manganese-catalyzed hydrogen transfer mediated acceptorless dehydrogenative coupling and borrowing hydrogenation reactions. ${ }^{4-53}$ Manganese catalyzed reformation leading to the generation of value-added products has started gathering keen interest. ${ }^{54-59}$ In our continuous quest to develop sustainable manganese-based catalytic systems, herein, we report homogeneous manganese(I)-complexes for the reformation of EG to highly demanded GA and LA molecules with the generation of pure hydrogen gas (Figure 1C). Encouragingly, the catalysts operate at a lower temperature and exhibit excellent yields and selectivities for GA and LA, suppressing all possible byproducts, with TON up to 2400 for GA (highest reported till date) and 11,500 for LA, respectively. To the best of our knowledge, manganese-catalyzed reformation of EG to GA and LA has not been reported thus far.

\section{RESULTS AND DISCUSSION}

Manganese-catalyzed reformation of EG to GA. For the reformation of EG to GA, we commenced our investigation by screening various bench stable manganese(I)-pincer-complexes Mn-I to Mn-VII as catalysts. The complexes have previously been utilized for diverse waste-free redox transformations. ${ }^{45-46,60-63}$ In a typical reaction, $5 \mathrm{mmol}$ of EG was treated with $0.5 \mathrm{~mol} \%$ of manganese(I)-complex in the presence of $\mathrm{KOH}$ in ${ }^{t} \mathrm{AmOH}$ at $140{ }^{\circ} \mathrm{C}$ (Scheme 1). Progress of the reaction was monitored by measuring the evolved hydrogen.

Scheme 1. Evaluation of Manganese(I)-Complexes for Reformation of EG to GA and Hydrogen. ${ }^{a}$

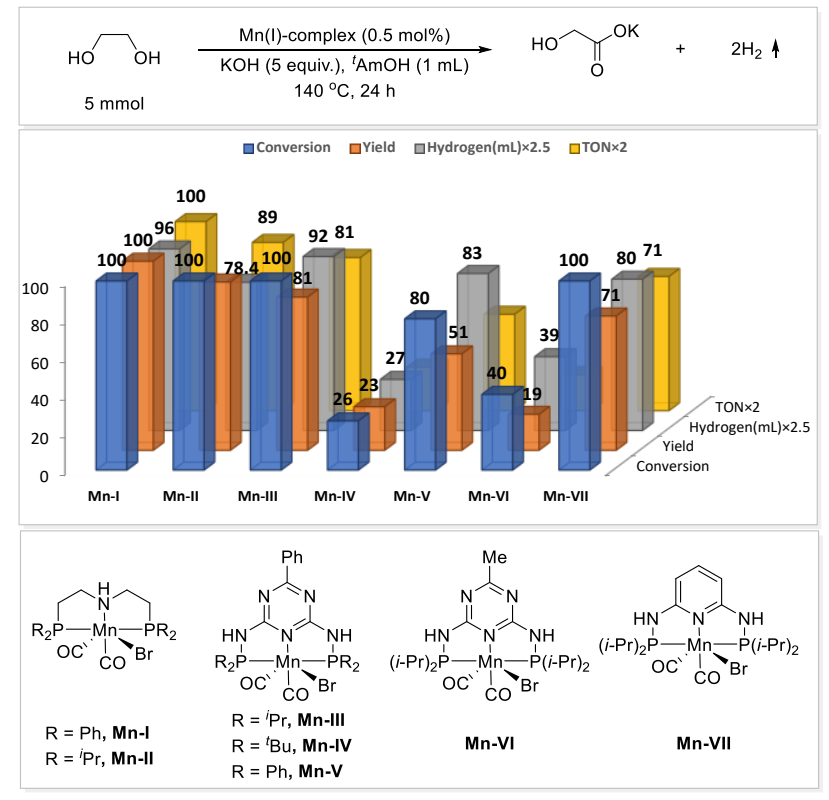

${ }^{a}$ Reaction conditions: EG (5 mmol), Mn(I)-complex (0.5 $\mathrm{mol} \%), \mathrm{KOH}(25 \mathrm{mmol}),{ }^{t} \mathrm{AmOH}(1 \mathrm{~mL})$ at $140{ }^{\circ} \mathrm{C}$ for $24 \mathrm{~h}$ under $\mathrm{Ar}$ in a $100 \mathrm{~mL}$ reaction tube with sidearm. Conversion, yield, and selectivity were determined by ${ }^{1} \mathrm{H}$ NMR analysis using sodium acetate as an internal standard. Hydrogen volume was measured using an inverted burette.
Much to our delight, among the tested Mn(I)-complexes, MnI, stabilized by the ${ }^{\mathrm{Ph}} \mathrm{MACHO}$ ligand, gave the highest volume of hydrogen $\left(240 \mathrm{~mL}\right.$, TON >198). ${ }^{1} \mathrm{H}$ NMR analysis of the reaction mixture indicated complete conversion of EG and exclusive formation of glycolate ( $>99 \%$ yield). Although MnII, Mn-III, and Mn-VII resulted in full conversion of EG, the selectivity for GA was measured to be slightly less (up to $89 \%$ yield and TON = 178). Complexes Mn-IV with a bulky ${ }^{t} \mathrm{Bu}$ phosphine ligand and Mn-VI were noticed to be less active for this reaction (up to $23 \%$ yield and $\mathrm{TON}=46$ ).

Encouraged by these promising results, we screened various reaction parameters to improve the reaction's efficiency (Table 1). Monitoring the reaction time indicated that the reaction was completed at 9h (Entries 1-3). The use of bases other than $\mathrm{KOH}$ was found to be detrimental to the reaction (entries 45 ). The equivalency of the base used was also crucial, and lowering the equivalency resulted in a decrease in the yield (entries 6-7). The reaction was susceptible to ${ }^{t} \mathrm{AmOH}$ as other mediums performed poorly (entries 8-9). Notably, the reaction was also found to proceed smoothly at $130{ }^{\circ} \mathrm{C}$ without compromising yield and selectivity (entry 10). Control experiments demonstrated the importance of the catalyst and base (entries 11-12). Further details of reaction optimization are tabulated in Tables S1-S5.

Table 1. Optimization Studies for the Mn-I Catalyzed Reformation of EG to GA. ${ }^{a}$

\begin{tabular}{|c|c|c|c|c|c|c|}
\hline Hó & $\mathrm{OH}$ & $\begin{array}{c}\text { Mn-l }(0.04-0 \\
\text { base, Sol } \\
130-140^{\circ} \mathrm{C},\end{array}$ & $\begin{array}{l}5 \mathrm{~mol} \%) \\
\text { vent } \\
6-24 \mathrm{~h}\end{array}$ & & $\prod_{0}$ & $2 \mathrm{H}_{2} \uparrow$ \\
\hline Entry & Solvent & Base ( $x$ equiv.) & Time (h) & Yield (GA) & Hydrogen $(\mathrm{mL})$ & TON (GA) \\
\hline 1 & ${ }^{t} \mathrm{AmOH}$ & $\mathrm{KOH}(5)$ & 24 & $>99 \%$ & 240 & $>198$ \\
\hline 2 & ${ }^{t} \mathrm{AmOH}$ & $\mathrm{KOH}(5)$ & 6 & $82 \%$ & 192 & 164 \\
\hline 3 & ${ }^{t} \mathrm{AmOH}$ & $\mathrm{KOH}(5)$ & 9 & $>99 \%$ & 230 & $>198$ \\
\hline 4 & ${ }^{t} \mathrm{AmOH}$ & $\mathrm{NaOH}(5)$ & 9 & $15 \%$ & 179 & 30 \\
\hline 5 & ${ }^{t} \mathrm{AmOH}$ & $\mathrm{Ba}(\mathrm{OH})_{2} \cdot 8 \mathrm{H}_{2} \mathrm{O}(5)$ & 9 & n.d. & n.d. & n.d. \\
\hline 6 & ${ }^{t} \mathrm{AmOH}$ & $\mathrm{KOH}(4)$ & 9 & $98 \%$ & 208 & 196 \\
\hline 7 & ${ }^{t} \mathrm{AmOH}$ & $\mathrm{KOH}(3)$ & 9 & $61 \%$ & 130 & 122 \\
\hline 8 & THF & $\mathrm{KOH}(5)$ & 9 & $10 \%$ & 25 & 20 \\
\hline 9 & toluene & $\mathrm{KOH}(5)$ & 9 & n.d. & n.d. & n.d. \\
\hline $10^{b}$ & ${ }^{t} \mathrm{AmOH}$ & $\mathrm{KOH}(5)$ & 9 & $>99 \%$ & 235 & $>198$ \\
\hline 11 & ${ }^{t} \mathrm{AmOH}$ & - & 9 & n.d. & n.d. & n.d. \\
\hline $12^{\circ}$ & ${ }^{t} \mathrm{AmOH}$ & $\mathrm{KOH}(5)$ & 9 & n.d. & n.d. & n.d. \\
\hline $13^{d}$ & ${ }^{t} \mathrm{AmOH}$ & KOH (5) & 12 & $96 \%$ & 925 & 2400 \\
\hline
\end{tabular}

${ }^{a}$ Reaction conditions: EG ( $\left.5 \mathrm{mmol}\right), \mathrm{Mn}-\mathrm{I}(0.5 \mathrm{~mol} \%)$, base, solvent $(1 \mathrm{~mL})$ at $140{ }^{\circ} \mathrm{C}$ for $6-24 \mathrm{~h}$ under $\mathrm{Ar}$ in a $100 \mathrm{~mL}$ reaction tube with a sidearm. Conversion, yield, and selectivity were determined by ${ }^{1} \mathrm{H}$ NMR analysis using sodium acetate as an internal standard. Hydrogen volume was measured using an inverted burette. ${ }^{b}$ At $130{ }^{\circ} \mathrm{C} .{ }^{c}$ No catalyst. ${ }^{d}$ EG (20 mmol), Mn-I (0.04 $\mathrm{mol} \%),{ }^{t} \mathrm{AmOH}(4 \mathrm{~mL})$ for $12 \mathrm{~h}$ at $140{ }^{\circ} \mathrm{C}$ under Ar. n.d. $=$ not determined.

Pleasingly, a scale-up reaction with $0.04 \mathrm{~mol} \%$ loading of Mn-I gave GA in $96 \%$ yield with the generation of $0.93 \mathrm{~L}$ of hydrogen reaching a TON of 2400 (Table 1, entry 13). To the best of our knowledge, it represents the highest TON for the EG reformation to GA reported to date using a molecularly defined metal complex. Notably, the gas chromatography analysis of the evolved gas showed that the pure hydrogen was evolved (SI, Section 11.13). It thus provides the possibility of being used as a clean energy source. Any further scale-up 
reaction was not performed due to the limitation of the reaction vessel.

Further, a catalyst recyclability test was performed to test the robustness of Mn-I in catalyzing the reformation of EG to GA and hydrogen (SI, section 6). The catalyst was found to be active for three cycles (on $10 \mathrm{mmol}$ of EG run), and a total of $1.3 \mathrm{~L}$ of hydrogen evolution was observed over a period of 72 h. After three cycles, the catalytic activity decreased.

Manganese-catalyzed dehydrogenative coupling of EG and MeOH for the synthesis of LA. We next extended the protocol towards synthesizing LA by the dehydrogenative coupling of $\mathrm{EG}$ and $\mathrm{MeOH}$. For the DHC reaction, $5 \mathrm{mmol}$ of EG was treated with $0.2 \mathrm{~mol} \%$ of the previously used manganese(I)-complexes and $\mathrm{KOH}$ in $\mathrm{MeOH}$ at $140{ }^{\circ} \mathrm{C}$ (Scheme 2). Gratifyingly, amongst the different manganese(I)-complexes tested, $\left({ }^{i \mathrm{Pr}} \mathrm{PN}{ }^{5} \mathrm{P}\right) \mathrm{Mn}(\mathrm{I})$-complex Mn-III with a triazine backbone gave a quantitative lactate yield $(\mathrm{TON}=495)$. Other tested manganese complexes Mn-I, Mn-II, and Mn-IV Mn-VII, which were efficient for EG reformation to GA, were found to be ineffective (TON up to 140) under these conditions, elaborating the need for appropriate catalyst design.

Scheme 2. Evaluation of Manganese(I)-Complexes for the Dehydrogenative Coupling of EG and MeOH to Yield LA. ${ }^{a}$

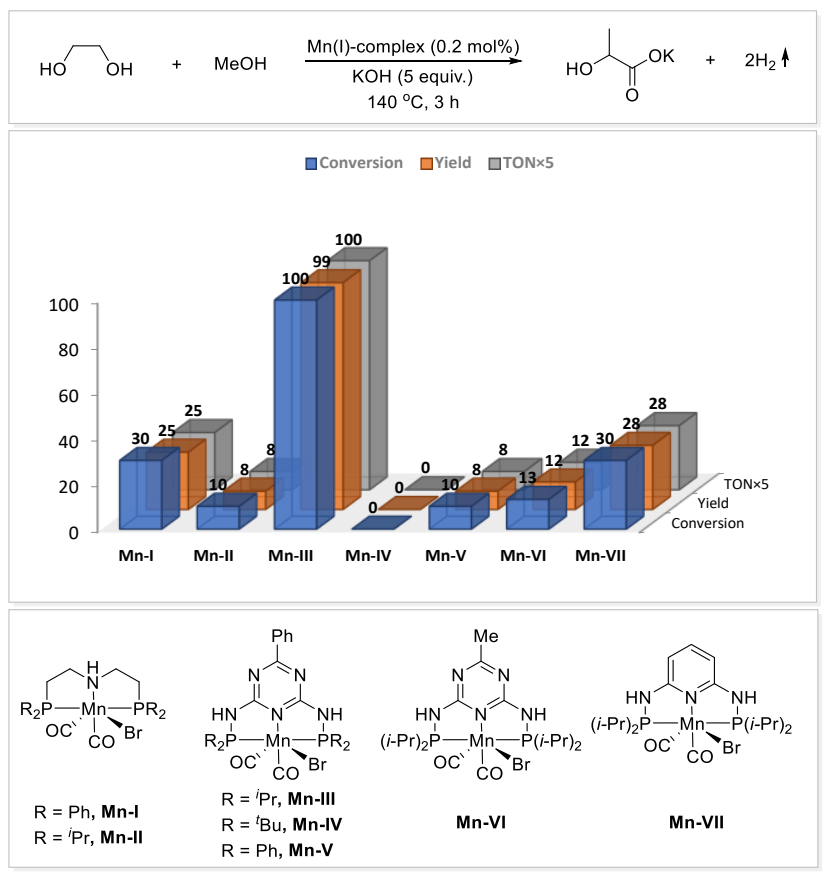

${ }^{a}$ Reaction conditions: EG (5 mmol), Mn(I)-complex $(0.2$ $\mathrm{mol} \%), \mathrm{KOH}(25 \mathrm{mmol}), \mathrm{MeOH}(4 \mathrm{~mL})$ at $140^{\circ} \mathrm{C}$ for $3 \mathrm{~h}$ under $\mathrm{Ar}$ in a $15 \mathrm{~mL}$ Schlenk tube. Conversion, yield, and selectivity were determined by ${ }^{1} \mathrm{H}$ NMR analysis using sodium acetate as an internal standard.

With the suitable manganese(I)-catalyst in hand, we then screen other reaction conditions to optimize the LA production (Table 2). The reaction was found to be sensitive to bases. Besides $\mathrm{KOH}$, other bases performed poorly under these conditions (entries 1-4). Lowering the equivalency of $\mathrm{KOH}$ also made the reaction sluggish and led to a slightly diminished yield of the desired product (entries 5-6). The MeOH equivalency was then varied. Notably, it was found that up to 5 equivalent of $\mathrm{MeOH}$ gives a quantitative yield of lactate (entries 7-9). A slightly lower conversion and yield were obtained when 2.5 equivalent of $\mathrm{MeOH}$ was used (entry 10). Controlled reactions in the absence of either the base or the catalyst resulted in no consumption of the EG, thereby stressing the need for both (entries 11-12).

Inspired by these promising results, we have lowered the catalyst concentration to boost the LA production. When a sixfold lower loading of Mn-I was used, a TON 3030 with a high turnover frequency TOF $=1010 \mathrm{~h}^{-1}$ was reached with maintaining high selectivity for LA (entry 13). Notably, lowering the catalyst loading to as low as $195 \mathrm{ppm}$ also gave the desired product in a high $97 \%$ yield with TON $=11,500$ within $72 \mathrm{~h}$ (entry 14).

Table 2. Optimization Studies for the Mn-III Catalyzed LA Synthesis via DHC of EG and MeOH. ${ }^{a}$

\begin{tabular}{|c|c|c|c|c|c|c|}
\hline $\mathrm{HO}$ & $\widehat{\mathrm{OH}}$ & Mn-III ( & $\begin{array}{c}\frac{(0.008-0}{\text { base }} \\
40^{\circ} \mathrm{C}, 3-\end{array}$ & $\underset{72 \mathrm{~h}}{\stackrel{2 \mathrm{~mol} \%)}{\longrightarrow}}$ & & $+2 \mathrm{H}_{2} \uparrow$ \\
\hline Entry & MeOH equiv. & Base (x equiv.) & Time (h) & Conversion (EG) & Yield (LA) & TON (LA) \\
\hline 1 & 20 & $\mathrm{KOH}(5)$ & 3 & $100 \%$ & $99 \%$ & 495 \\
\hline 2 & 20 & $\mathrm{NaOH}(5)$ & 3 & $6 \%$ & $4 \%$ & 20 \\
\hline 3 & 20 & $\mathrm{Ca}(\mathrm{OH})_{2}(5)$ & 3 & $16 \%$ & $5 \%$ & 25 \\
\hline 4 & 20 & $\mathrm{Ba}(\mathrm{OH})_{2} .8 \mathrm{H}_{2} \mathrm{O}(5)$ & 3 & n.d. & n.d. & n.d. \\
\hline 5 & 20 & $\mathrm{KOH}(4)$ & 3 & $87 \%$ & $81 \%$ & 405 \\
\hline 6 & 20 & $\mathrm{KOH}(3)$ & 3 & $85 \%$ & $76 \%$ & 380 \\
\hline 7 & 15 & $\mathrm{KOH}(5)$ & 3 & $100 \%$ & $99 \%$ & 495 \\
\hline 8 & 10 & $\mathrm{KOH}(5)$ & 3 & $100 \%$ & $99 \%$ & 495 \\
\hline 9 & 5 & $\mathrm{KOH}(5)$ & 3 & $100 \%$ & $99 \%$ & 495 \\
\hline 10 & 2.5 & $\mathrm{KOH}(5)$ & 3 & $96 \%$ & $77 \%$ & 375 \\
\hline 11 & 5 & - & 3 & n.d. & n.d. & n.d. \\
\hline $12^{b}$ & 5 & $\mathrm{KOH}(5)$ & 3 & n.d. & n.d. & n.d. \\
\hline $13^{c}$ & 5 & $\mathrm{KOH}(5)$ & 3 & $100 \%$ & $99 \%$ & 3030 \\
\hline $14^{d}$ & 5 & KOH (5) & 72 & $93 \%$ & $92 \%$ & 11,500 \\
\hline
\end{tabular}

${ }^{a}$ Reaction conditions: EG ( $\left.5 \mathrm{mmol}\right), \mathbf{M n}-\mathbf{I I I}(0.2 \mathrm{~mol} \%), \mathrm{KOH}$ ( $25 \mathrm{mmol}), \mathrm{MeOH}(1 \mathrm{~mL})$ for $3 \mathrm{~h}$ at $140{ }^{\circ} \mathrm{C}$ under Ar. Conversion, yield, and selectivity were determined by ${ }^{1} \mathrm{H}$ NMR analysis using sodium acetate as an internal standard. ${ }^{b}$ No catalyst, ${ }^{c}$ MnIII (0.033 mol\%). ${ }^{d}$ EG (20 mmol), Mn-III (0.008 mol\%, 195 $\mathrm{ppm}), \mathrm{MeOH}(4 \mathrm{~mL})$ for $72 \mathrm{~h}$.

Mechanistic studies. A plausible working model for the reformation of EG to GA and LA with pure hydrogen production is depicted in Scheme 3A. ${ }^{24,32}$ Ethylene glycol dehydrogenation occurs in the presence of manganese catalysts leading to the formation of glycolaldehyde with the liberation of hydrogen. In the absence of methanol, glycolaldehyde will undergo another cycle of dehydrogenation, leading to glyoxal formation. Subsequently, intramolecular Cannizzaro reaction of the in-situ generated glyoxal under basic conditions leads to the formation of the glycolate product. Alternatively, glycolaldehyde could also be converted to glycolate via an intermolecular Cannizzaro reaction.

A set of control and mechanistic experiments were then carried out to get inside the reformation reaction. Initially, several ${ }^{31} \mathrm{P}$ NMR experiments were performed to elucidate the involvement of relevant manganese intermediates in the reaction (Scheme 3B, SI Section 11.3). Treatment of the manganese precatalyst, Mn-I with base leads to the formation of Mn(I)-amido complex Mn-Ia following dehydrobromination. ${ }^{64} \mathbf{M n}$-Ia on treatment with excess water and ethylene 
Scheme 3. A Plausible Mechanism for Reformation of Ethylene Glycol to Glycolic acid and Lactic Acid and Mechanistic Studies.

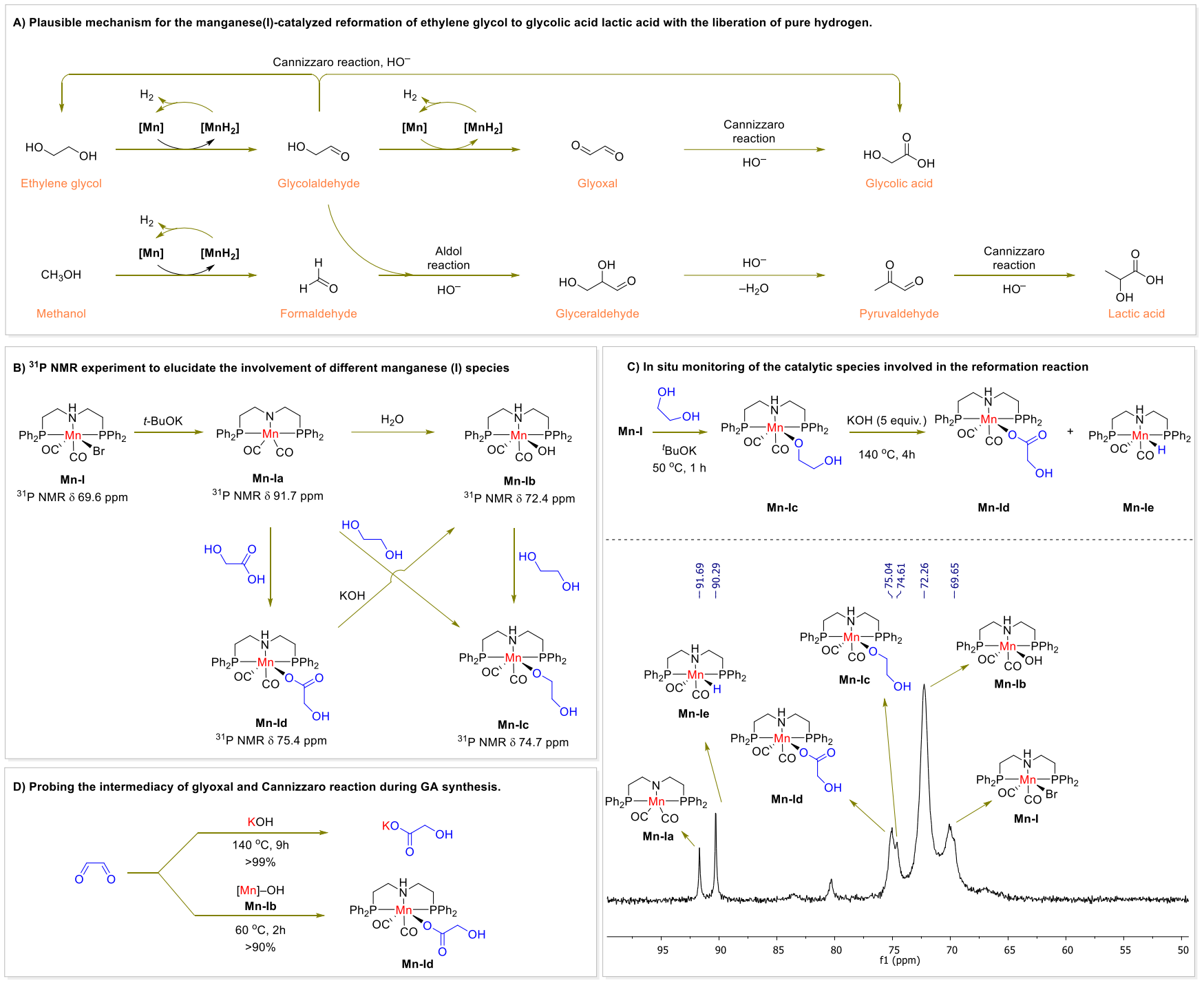

glycol forms the Mn(I)-hydroxy complex Mn-Ib and the Mn(I)-alkoxy complex Mn-Ic, respectively. The synthesis of the complexes similar to Mn-Ib,c have previously been reported. ${ }^{65}$ Again, the alkoxy complex Mn-Ic was formed when the hydroxide Mn-Ib was reacted with excess ethylene glycol. Additionally, the treatment of Mn-Ia with GA leads to the formation of Mn(I)-glycolato complex Mn-Id. When treated with an excess amount of $\mathrm{KOH}$, the latter liberates the hydroxide complex Mn-Ib and potassium glycolate.

We believe that the ethylene glycol dehydrogenation reaction occurred via the intermediacy of the alkoxy intermediate MnIc, formed directly from the amido complex Mn-1a or via the hydroxide Mn-1b (Scheme 3B). To elucidate the involvement of the alkoxy complex Mn-Ic, we have generated it in situ from Mn-I by treating it with base and EG. When thus formed, Mn-Ic was reacted with an excess $\mathrm{KOH}$ at $140{ }^{\circ} \mathrm{C}$ for $4 \mathrm{~h}$, we have observed the formation of manganese(I)-glycolate Mn-Id and the manganese(I)-hydride Mn-Ie along with the intermediates Mn-1a,b (Scheme 3C). Additionally, the above experiments (Scheme 3B,C) highlight the existence of the complexes Mn-Ia-e in solution during the catalysis.

Furthermore, the alkoxy complex Mn-Ic was synthesized separately and used for catalytic and stoichiometric EG reformation reactions (SI, Section 11.8-11.10). Under these conditions, $95 \%$ and $93 \%$ yields of GA, respectively, were obtained. It further suggested the intermediacy of Mn-Ic. Moreover, independently synthesized the manganese(I)-hydroxide Mn-Ib could also catalyze the reformation reaction in similar efficiencies (71\% yield, SI section 11.12), suggesting its intermediacy during the reaction.

Kinetics studies for the Mn-I catalyzed EG reformation to GA were then performed to shed more light on the mechanism (See SI, Section 11.6). The hydrogen evolution over time was measured using an inverted burette to determine the initial rates. For the reaction performed at $[\mathrm{EG}]=1.67 \mathrm{M},[\mathrm{KOH}]=$ $8.33 \mathrm{M}$, the initial rate for the hydrogen evolution was found to increase linearly with the increasing concentration of Mn-I (0.005-0.01 M), indicating the first-order kinetics. Different initial concentrations of EG (1.67-6.67 M) were noticed to impart minimal effect on the reaction rate. This suggested that the Mn-I catalyzed dehydrogenation of ethylene glycol is a fast and facile step and might not contribute to the overall 
reaction rate. Notably, when the reaction was conducted with the varying initial concentration of $\mathrm{KOH}(8.33-13.3 \mathrm{M})$, the reaction was found to be second-order with $[\mathrm{KOH}]$. This indicated the executive role of the base during the ethylene glycol dehydrogenation and Cannizzaro reaction.

To probe the intermediacy of glyoxal and base-mediated Cannizzaro reaction, we have studied the reactions of glyoxal with $\mathrm{KOH}$, and $99 \%$ yield of potassium glycolate was observed in 9h (Scheme 3D, SI, section 11.1). A similar reaction, when performed with the $\mathrm{Mn}(\mathrm{I})$-hydroxide $\mathbf{M n - 1} \mathbf{b}$, formation of manganese glycolate Mn-Id, was noticed in $>90 \%$ yield (Scheme 3D, SI section 11.4.3). During the catalysis, Mn-Id

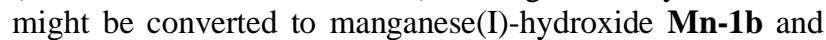
potassium glycolate in the presence of $\mathrm{KOH}$ to close the cycle. The conversion of Mn-Id to Mn-1b and potassium glycolate was observed as discussed earlier (Scheme 3B).

Furthermore, the EG reformation reaction rates were measured at different reaction temperatures $\left(120\right.$ to $140{ }^{\circ} \mathrm{C}$, SI Section 11.7). From the Eyring analysis, we have calculated overall barrier $\Delta \mathrm{G}^{\$}(298)=103.7 \mathrm{~kJ} \mathrm{~mol}^{-1}$ with $\Delta \mathrm{H}^{\ddagger}=+62.4 \mathrm{~kJ} \mathrm{~mol}^{-}$ ${ }^{1}$ and $\Delta \mathrm{S}^{\dagger}=-138.6 \mathrm{~J} \mathrm{~mol}^{-1} \mathrm{~K}^{-1}$. The negative value of entropy of activation suggested the involvement of the associative pathway.

During the LA synthesis via DHC reaction in the presence of $\mathrm{MeOH}$, both $\mathrm{EG}$ and $\mathrm{MeOH}$ undergo dehydrogenation to the glycolaldehyde and formaldehyde, respectively, with the liberation of hydrogen (Scheme 3A). In this regard, Mn-III mediated dehydrogenation of $\mathrm{MeOH}$ to formaldehyde has previously been reported. ${ }^{61,66} \mathrm{~A}$ cross-aldol condensation of in situ generated glycolaldehyde and formaldehyde in the presence of a base gives glyceraldehyde. Subsequent dehydration of the glyceraldehyde results in pyruvaldehyde, which undergoes intramolecular Cannizzaro reaction under alkaline conditions, leading to the formation of LA.

\section{CONCLUSION}

A sustainable approach to synthesizing two valuable platform molecules, glycolic acid, and lactic acid, from readily available renewable resource ethylene glycol has been disclosed. The reformation has been carried out using Earth-abundant transition metal catalysts. A high TON and selectivity for the synthesis of both GA and LA were obtained. Pure hydrogen gas is liberated during the acceptorless reformation process. As a future outlook, we believe that this report will open a new avenue towards sustainably obtaining value-added products and serve as a milestone towards making synthetic chemistry more environment-friendly.

\section{ASSOCIATED CONTENT}

\section{Supporting Information}

The Supporting Information is available free of charge on the ACS Publications website.

Experimental procedures, analytical and kinetic data, NMR spec$\operatorname{tra}(\mathrm{pdf}))$

\section{AUTHOR INFORMATION}

\section{Corresponding Author}

Biplab Maji - Department of Chemical Sciences, Indian Institute of Science Education and Research Kolkata, Mohanpur 741246, India

orcid.org/0000-0001-5034-423X

Email: bm@iiserkol.ac.in

\section{Author}

Satyadeep Waiba - Department of Chemical Sciences, Indian Institute of Science Education and Research Kolkata, Mohanpur 741246, India

\section{Author Contributions}

All authors have given approval to the final version of the manuscript.

Notes

The authors declare no competing financial interest.

\section{ACKNOWLEDGMENT}

The author thanks IISER Kolkata and CSIR 02(0405)/21/EMRII for financial support. S. W. thanks IISER Kolkata for funding and research facility. The author also thanks Miss Soumi Chakraborty and Prof. Swadhin K. Mandal for the gas chromatography experiment.

\section{REFERENCES}

1. Centi, G.; Santen, v., R.A. Catalysis for renewables : from feedstock to energy production. Wiley-VCH: Weinheim, 2007.

2. Trost, B. The atom economy--a search for synthetic efficiency. Science 1991, 254 (5037), 1471-1477.

3. Newhouse, T.; Baran, P. S.; Hoffmann, R. W. The economies of synthesis. Chem. Soc. Rev. 2009, 38 (11), 3010-3021.

4. Goldstein, I. S. Organic Chemicals from Biomass. CRC Press: 1981; p 144.

5. Kersten, S.; Van Swaaij, W.; Lefferts, L.; Seshan, K., Catalysis for renewables: from feedstock to energy production. 2007.

6. Ragauskas, A. J.; Williams, C. K.; Davison, B. H.; Britovsek, G.; Cairney, J.; Eckert, C. A.; Frederick, W. J.; Hallett, J. P.; Leak, D. J.; Liotta, C. L.; Mielenz, J. R.; Murphy, R.; Templer, R.; Tschaplinski, T. The Path Forward for Biofuels and Biomaterials. Science 2006, 311 (5760), 484-489.

7. Li, C.; Zhao, X.; Wang, A.; Huber, G. W.; Zhang, T. Catalytic Transformation of Lignin for the Production of Chemicals and Fuels. Chem. Rev. 2015, 115 (21), 11559-11624.

8. Klass, D. L. Biomass for renewable energy, fuels, and chemicals. Elsevier: 1998

9. Yue, H.; Zhao, Y.; Ma, X.; Gong, J. Ethylene glycol: properties, synthesis, and applications. Chem. Soc. Rev. 2012, 41 (11), 4218-4244.

10. Wang, A.; Zhang, T. One-Pot Conversion of Cellulose to Ethylene Glycol with Multifunctional Tungsten-Based Catalysts. Acc. Chem. Res. 2013, 46 (7), 1377-1386.

11. Zou, Y.-Q.; von Wolff, N.; Anaby, A.; Xie, Y.; Milstein, D. Ethylene glycol as an efficient and reversible liquid-organic hydrogen carrier. Nat. Catal. 2019, 2 (5), 415-422.

12. J. Kubitschke, H. L., H. Strutz Carboxylic Acids, Aliphatic in Ullmann's Encyclopedia of Industrial Chemistry. Wiley-VCH: Weinheim, 2014.

13. Holm, M. S.; Saravanamurugan, S.; Taarning, E. Conversion of Sugars to Lactic Acid Derivatives Using Heterogeneous Zeotype Catalysts. Science 2010, 328 (5978), 602605.

14. Dusselier, M.; Van Wouwe, P.; Dewaele, A.; Makshina, E.; Sels, B. F. Lactic acid as a platform chemical in the biobased 
economy: the role of chemocatalysis. Energy Environ. Sci. 2013, 6 (5), 1415-1442.

15. Castillo Martinez, F. A.; Balciunas, E. M.; Salgado, J. M.; Domínguez González, J. M.; Converti, A.; Oliveira, R. P. d. S. Lactic acid properties, applications and production: A review. Trends Food Sci. Technol. 2013, 30 (1), 70-83.

16. Wang, Y. Bioadaptability: An Innovative Concept for Biomaterials. J. Mater. Sci. Technol. 2016, 32 (9), 801-809.

17. Gentile, P.; Chiono, V.; Carmagnola, I.; Hatton, P. V. An Overview of Poly(lactic-co-glycolic) Acid (PLGA)-Based Biomaterials for Bone Tissue Engineering. Int. J. Mol. Sci. 2014, 15 (3), 3640-3659

18. Glycolic Acid Market-Forecasts from 2018 to 2023. https://www.researchandmarkets.com/reports/4542547/glycolicacid-market-forecasts.

19. Lee, S. Y.; Kim, H. U.; Chae, T. U.; Cho, J. S.; Kim, J. W.; Shin, J. H.; Kim, D. I.; Ko, Y.-S.; Jang, W. D.; Jang, Y.-S. A comprehensive metabolic map for production of bio-based chemicals. Nat. Catal. 2019, 2 (1), 18-33.

20. Prager, L.; Dowideit, P.; Langguth, H.; Schuchmann, H.P.; von Sonntag, C. Hydrolytic removal of the chlorinated products from the oxidative free-radical-induced degradation of chloroethylenes: acid chlorides and chlorinated acetic acids. J. Chem. Soc., Perkin Trans. 2 2001, (9), 1641-1647.

21. Zou, Y.-Q.; von Wolff, N.; Rauch, M.; Feller, M.; Zhou, Q.-Q.; Anaby, A.; Diskin-Posner, Y.; Shimon, L. J. W.; Avram, L.; Ben-David, Y.; Milstein, D. Homogeneous Reforming of Aqueous Ethylene Glycol to Glycolic Acid and Pure Hydrogen Catalyzed by Pincer-Ruthenium Complexes Capable of Metal-Ligand Cooperation. Chem. Eur. J. 2021, 27 (14), 4715-4722.

22. Trincado, M.; Kühlein, K.; Grützmacher, H. MetalLigand Cooperation in the Catalytic Dehydrogenative Coupling (DHC) of Polyalcohols to Carboxylic Acid Derivatives. Chem. Eur. J. 2011, 17 (42), 11905-11913.

23. van Haasterecht, T.; van Deelen, T. W.; de Jong, K. P.; Bitter, J. H. Transformations of polyols to organic acids and hydrogen in aqueous alkaline media. Catal. Sci. Tech. 2014, 4 (8), 2353-2366.

24. Zhan, Y.; Hou, W.; Li, G.; Shen, Y.; Zhang, Y.; Tang, Y. Oxidant-Free Transformation of Ethylene Glycol toward Glycolic Acid in Water. ACS Sustainable Chem. Eng. 2019, 7 (21), 1755917564.

25. Wu, J.; Shen, L.; Chen, Z.-N.; Zheng, Q.; Xu, X.; Tu, T. Iridium-Catalyzed Selective Cross-Coupling of Ethylene Glycol and Methanol to Lactic Acid. Angew. Chem. Int. Ed. 2020, 59 (26), 10421-10425.

26. Cortright, R. D.; Davda, R. R.; Dumesic, J. A. Hydrogen from catalytic reforming of biomass-derived hydrocarbons in liquid water. Nature 2002, 418 (6901), 964-967.

27. Huber, G. W.; Shabaker, J. W.; Dumesic, J. A. Raney Ni$\mathrm{Sn}$ Catalyst for $\mathrm{H}_{2}$ Production from Biomass-Derived Hydrocarbons. Science 2003, 300 (5628), 2075-2077.

28. Morton, D.; Cole-Hamilton, D. J. Molecular hydrogen complexes in catalysis: highly efficient hydrogen production from alcoholic substrates catalysed by ruthenium complexes. J. Chem. Soc. Chem. Commun. 1988, (17), 1154-1156.

29. Li, Y.; Nielsen, M.; Li, B.; Dixneuf, P. H.; Junge, H.; Beller, M. Ruthenium-catalyzed hydrogen generation from glycerol and selective synthesis of lactic acid. Green Chem. 2015, 17 (1), 193 198.

30. Manas, M. G.; Campos, J.; Sharninghausen, L. S.; Lin, E.; Crabtree, R. H. Selective catalytic oxidation of sugar alcohols to lactic acid. Green Chem. 2015, 17 (1), 594-600.

31. Lu, Z.; Demianets, I.; Hamze, R.; Terrile, N. J.; Williams, T. J. A Prolific Catalyst for Selective Conversion of Neat Glycerol to Lactic Acid. ACS Catal. 2016, 6 (3), 2014-2017.
32. Sharninghausen, L. S.; Campos, J.; Manas, M. G.; Crabtree, R. H. Efficient selective and atom economic catalytic conversion of glycerol to lactic acid. Nat. Commun. 2014, 5 (1), 5084.

33. Sharninghausen, L. S.; Mercado, B. Q.; Crabtree, R. H.; Hazari, N. Selective conversion of glycerol to lactic acid with iron pincer precatalysts. Chem. Commun. 2015, 51 (90), 16201-16204.

34. Sun, Z.; Liu, Y.; Chen, J.; Huang, C.; Tu, T. Robust Iridium Coordination Polymers: Highly Selective, Efficient, and Recyclable Catalysts for Oxidative Conversion of Glycerol to Potassium Lactate with Dihydrogen Liberation. ACS Catal. 2015, 5 (11), 6573-6578

35. Maji, B.; Barman, M. K. Recent Developments of Manganese Complexes for Catalytic Hydrogenation and Dehydrogenation Reactions. Synthesis 2017, 49 (15), 3377-3393.

36. Gorgas, N.; Kirchner, K. Isoelectronic Manganese and Iron Hydrogenation/Dehydrogenation Catalysts: Similarities and Divergences. Acc. Chem. Res. 2018, 51 (6), 1558-1569.

37. Mukherjee, A.; Milstein, D. Homogeneous Catalysis by Cobalt and Manganese Pincer Complexes. ACS Catal. 2018, 8 (12), 11435-11469.

38. $\mathrm{Hu}, \mathrm{Y}$; Wang, C. Manganese-Catalyzed C-H Olefination Reactions. ChemCatChem 2019, 11 (4), 1167-1174.

39. Waiba, S.; Maji, B. Manganese Catalyzed Acceptorless Dehydrogenative Coupling Reactions. ChemCatChem 2020, 12 (7), 1891-1902.

40. Das, K.; Barman, M. K.; Maji, B. Advancements in multifunctional manganese complexes for catalytic hydrogen transfer reactions. Chem. Commun. 2021.

41. Maji, M.; Panja, D.; Borthakur, I.; Kundu, S. Recent advances in sustainable synthesis of N-heterocycles following acceptorless dehydrogenative coupling protocol using alcohols. $\mathrm{Org}$. Chem. Front 2021, 8 (11), 2673-2709.

42. Wang, Y.; Wang, M.; Li, Y.; Liu, Q. Homogeneous manganese-catalyzed hydrogenation and dehydrogenation reactions. Chem 2021, 7 (5), 1180-1223.

43. Filonenko, G. A.; van Putten, R.; Hensen, E. J. M.; Pidko, E. A. Catalytic (de)hydrogenation promoted by non-precious metals - Co, Fe and Mn: recent advances in an emerging field. Chem. Soc. Rev. 2018, 47 (4), 1459-1483.

44. Chakraborty, S.; Das, U. K.; Ben-David, Y.; Milstein, D. Manganese Catalyzed $\alpha$-Olefination of Nitriles by Primary Alcohols. J. Am. Chem. Soc. 2017, 139 (34), 11710-11713.

45. Peña-López, M.; Piehl, P.; Elangovan, S.; Neumann, H.; Beller, M. Manganese-Catalyzed Hydrogen-Autotransfer $\mathrm{C}-\mathrm{C}$ Bond Formation: $\alpha$-Alkylation of Ketones with Primary Alcohols. Angew. Chem. Int. Ed. 2016, 55 (48), 14967-14971.

46. Mukherjee, A.; Nerush, A.; Leitus, G.; Shimon, L. J. W.; Ben David, Y.; Espinosa Jalapa, N. A.; Milstein, D. ManganeseCatalyzed Environmentally Benign Dehydrogenative Coupling of Alcohols and Amines to Form Aldimines and H2: A Catalytic and Mechanistic Study. J. Am. Chem. Soc. 2016, 138 (13), 4298-4301.

47. Elangovan, S.; Neumann, J.; Sortais, J.-B.; Junge, K.; Darcel, C.; Beller, M. Efficient and selective $\mathrm{N}$-alkylation of amines with alcohols catalysed by manganese pincer complexes. Nat. Commun. 2016, 7, 12641

48. Barman, M. K.; Waiba, S.; Maji, B. Manganese-Catalyzed Direct Olefination of Methyl-Substituted Heteroarenes with Primary Alcohols. Angew. Chem. Int. Ed. 2018, 57 (29), 9126-9130.

49. Jana, A.; Reddy, C. B.; Maji, B. Manganese Catalyzed $\alpha-$ Alkylation of Nitriles with Primary Alcohols. ACS Catal. 2018, 8 (10), 9226-9231.

50. Das, K.; Kumar, A.; Jana, A.; Maji, B. Synthesis and characterization of $\mathrm{N}, \mathrm{N}$-chelate manganese complexes and applications in $\mathrm{CN}$ coupling reactions. Inorg. Chim. Acta 2020, 502, 119358

51. Jana, A.; Das, K.; Kundu, A.; Thorve, P. R.; Adhikari, D.; Maji, B. A Phosphine-Free Manganese Catalyst Enables 
Stereoselective Synthesis of $(1+\mathrm{n})$-Membered Cycloalkanes from Methyl Ketones and 1,n-Diols. ACS Catal. 2020, 10 (4), 2615-2626.

52. Waiba, S.; Jana, S. K.; Jati, A.; Jana, A.; Maji, B. Manganese complex-catalysed $\alpha$-alkylation of ketones with secondary alcohols enables the synthesis of $\beta$-branched carbonyl compounds. Chem. Commun. 2020, 56 (60), 8376-8379.

53. Fu, S.; Shao, Z.; Wang, Y.; Liu, Q. Manganese-Catalyzed Upgrading of Ethanol into 1-Butanol. J. Am. Chem. Soc. 2017, 139 (34), 11941-11948.

54. Kar, S.; Goeppert, A.; Kothandaraman, J.; Prakash, G. K. S. Manganese-Catalyzed Sequential Hydrogenation of $\mathrm{CO} 2$ to Methanol via Formamide. ACS Catal. 2017, 7 (9), 6347-6351.

55. Ryabchuk, P.; Stier, K.; Junge, K.; Checinski, M. P.; Beller, M. Molecularly Defined Manganese Catalyst for LowTemperature Hydrogenation of Carbon Monoxide to Methanol. $J$. Am. Chem. Soc. 2019, 141 (42), 16923-16929.

56. Kaithal, A.; Werlé, C.; Leitner, W. Alcohol-Assisted Hydrogenation of Carbon Monoxide to Methanol Using Molecular Manganese Catalysts. JACS Au 2021, 1 (2), 130-136.

57. Kaithal, A.; Hölscher, M.; Leitner, W. Carbon monoxide and hydrogen (syngas) as a C1-building block for selective catalytic methylation. Chem. Sci. 2021, 12 (3), 976-982.

58. Kaithal, A.; Chatterjee, B.; Werle, C.; Leitner, W. Acceptorless dehydrogenation of methanol to carbon monoxide and hydrogen using molecular catalysts. Angew. Chem. Int. Ed. 2021, n/a (n/a).

59. Andérez-Fernández, M.; Vogt, L. K.; Fischer, S.; Zhou, W.; Jiao, H.; Garbe, M.; Elangovan, S.; Junge, K.; Junge, H.; Ludwig, R.; Beller, M. A Stable Manganese Pincer Catalyst for the Selective Dehydrogenation of Methanol. Angew. Chem. Int. Ed. 2017, 56 (2), 559-562.

60. Mastalir, M.; Glatz, M.; Pittenauer, E.; Allmaier, G.; Kirchner, K. Sustainable Synthesis of Quinolines and Pyrimidines Catalyzed by Manganese PNP Pincer Complexes. J. Am. Chem. Soc. 2016, 138 (48), 15543-15546.

61. Deibl, N.; Kempe, R. Manganese-Catalyzed Multicomponent Synthesis of Pyrimidines from Alcohols and Amidines. Angew. Chem. Int. Ed. 2017, 56 (6), 1663-1666.

62. Garbe, M.; Junge, K.; Beller, M. Homogeneous Catalysis by Manganese-Based Pincer Complexes. Eur. J. Org. Chem. 2017, 2017 (30), 4344-4362.

63. Kallmeier, F.; Dudziec, B.; Irrgang, T.; Kempe, R. Manganese-Catalyzed Sustainable Synthesis of Pyrroles from Alcohols and Amino Alcohols. Angew. Chem. Int. Ed. 2017, 56 (25), 7261-7265.

64. Wang, Y.; Zhu, L.; Shao, Z.; Li, G.; Lan, Y.; Liu, Q. Unmasking the Ligand Effect in Manganese-Catalyzed Hydrogenation: Mechanistic Insight and Catalytic Application. $J$. Am. Chem. Soc. 2019, 141 (43), 17337-17349.

65. Shao, Z.; Wang, Y.; Liu, Y.; Wang, Q.; Fu, X.; Liu, Q. A general and efficient Mn-catalyzed acceptorless dehydrogenative coupling of alcohols with hydroxides into carboxylates. Org. Chem. Front 2018, 5 (8), 1248-1256.

66. Schlagbauer, M.; Kallmeier, F.; Irrgang, T.; Kempe, R. Manganese-Catalyzed $\beta$-Methylation of Alcohols by Methanol. Angew. Chem. Int. Ed. 2020, 59 (4), 1485-1490. 